Prepared for the U.S. Department of Energy under Contract DE-AC05-76RL01830

\title{
Geochemical Study of a Grab Sample Collected From 216 B-55 Crib in the 200-SC-1 Operable Unit
}

Michael Lindberg

JULY 2008

Pacific Northwest

NATIONAL LABORATORY 
07/09/08 11:38

To: Dana Widrig

From: Michael J. Lindberg

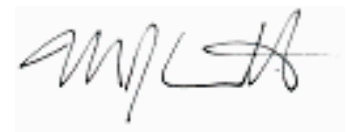

Environmental Sciences Laboratory

Energy and Environment Directorate, Pacific Northwest National Laboratory

Subject: Geochemical Study of a Grab Sample Collected From 216 B-55 Crib in the 200-SC-1 Operable Unit, Sample Delivery Group ESL080024, SAF Number F08-031

This letter contains the following information for sample delivery group ESL080024

- Cover Sheet

- Narrative

- Analytical Results

- Quality Control

- Chain of Custodies 


\section{Introduction}

On May 15, 2008 a grab sample was received from 216 B-55 Crib in the 200-SC-1 Operable Unit for geochemical studies.

\section{Analytical Results/Methodology}

The analyses for this project were performed at the 325 building located in the 300 Area of the Hanford Site. The analyses were performed according to Pacific Northwest National Laboratory (PNNL) approved procedures and/or nationally recognized test procedures. The data sets include the sample identification numbers, analytical results, estimated quantification limits (EQL), and quality control data.

\section{Quality Control}

The preparatory and analytical quality control requirements, calibration requirements, acceptance criteria, and failure actions are defined in the on-line QA plan “Conducting Analytical Work in Support of Regulatory Programs” (CAW). This QA plan implements the Hanford Analytical Services Quality Assurance Requirements Documents (HASQARD) for PNNL.

\section{Definitions}

Dup Duplicate

RPD Relative Percent Difference

\section{Sample Receipt}

The sample was received with a chain of custody (COC) and was analyzed according to the sample identification number supplied by the client. The sample was refrigerated upon receipt until prepared for analysis.

The sample was received with custody seals intact unless noted in the Case Narrative.

\section{Holding Times}

Holding time is defined as the time from sample preparation to the time of analyses. The prescribed holding times were met for all analytes unless noted in the Case Narrative.

\section{Analytical Results}

All reported analytical results meet the requirements of the CAW or client specified SOW unless noted in the case narrative. 


\section{Case Narrative Report}

Test reports/summary forms for blank samples

No Discrepancies Noted

Laboratory control samples (LCS):

No Discrepancies Noted

Post spike (PS) and post spike duplicate (PSD) data

No Discrepancies Noted

Analytical duplicate data

No Discrepancies Noted

\section{Hold Time Report}

No Discrepancies Noted

\section{DISCLAIMER}

This report was prepared as an account of work sponsored by an agency of the United States Government. Neither the United States Government nor any agency thereof, nor Battelle Memorial Institute, nor any of their employees, makes any warranty, express or implied, or assumes any legal liability or responsibility for the accuracy, completeness, or usefulness of any information, apparatus, product, or process disclosed, or represents that its use would not infringe privately owned rights. Reference herein to any specific commercial product, process, or service by trade name, trademark, manufacturer, or otherwise does not necessarily constitute or imply its endorsement, recommendation, or favoring by the United States Government or any agency thereof, or Battelle Memorial Institute. The views and opinions of authors expressed herein do not necessarily state or reflect those of the United States Government or any agency thereof.

\section{SAMPLES INCLUDED IN THIS REPORT}

\section{6-B-55 C6743}

HEIS No.

B1VDW3

$\begin{array}{ll}\text { Laboratory ID } & \text { Matrix } \\ 0806012-01 & \text { SOIL }\end{array}$

\author{
Date Collected \\ 5/13/08 07:35
}

Date Received

5/15/08 12:50 
The following analyses were performed on the following samples included in this report:

1:1 DI Water Extract

Anions By Ion Chromatography

Moisture Content

Percent Solids

\section{SAMPLES ANALYZED IN THIS REPORT}

HEIS No.

B1VDW3

\begin{abstract}
Laboratory ID Matrix
0806012-01 SOIL
\end{abstract}

Date Collected

5/13/08 07:35
Date Received

5/15/08 12:50 


\section{Wet Chemistry}

Moisture Content (\% by Weight) by AGG-WC-001

Lab ID HEIS No. Results

EQL Analyzed

Batch

0806012-01

B1VDW3

3.01

N/A

$6 / 19 / 08$

$8 \mathrm{~F} 13002$ 


\section{Anions by Ion Chromatography}

\begin{tabular}{|c|c|c|c|c|c|c|c|}
\hline CAS \# & Analyte & Results & Units & EQL & Analyzed & Batch & Method \\
\hline HEIS No. & B1VDW3 & \multicolumn{3}{|c|}{ Lab ID: $\quad 0806012-01$} & & & \\
\hline $16984-48-8$ & Fluoride & $<2.07$ & ug/g dry & 2.07 & $6 / 19 / 08$ & 8F17009 & AGG-IC-001 \\
\hline $16887-00-6$ & Chloride & $<5.16$ & ug/g dry & 5.16 & 6/19/08 & 8F17009 & AGG-IC-001 \\
\hline $14797-65-0$ & Nitrite & $<10.3$ & ug/g dry & 10.3 & 6/19/08 & 8F17009 & AGG-IC-001 \\
\hline 24959-67-9 & Bromide & $<10.3$ & ug/g dry & 10.3 & 6/19/08 & 8F17009 & AGG-IC-001 \\
\hline $14797-55-8$ & Nitrate & $<10.3$ & ug/g dry & 10.3 & 6/19/08 & $8 F 17009$ & AGG-IC-001 \\
\hline $14808-79-8$ & Sulfate & 44.5 & ug/g dry & 15.5 & 6/19/08 & 8F17009 & AGG-IC-001 \\
\hline 14265-44-2 & Phosphate & $<15.5$ & ug/g dry & 15.5 & 6/19/08 & 8F17009 & AGG-IC-001 \\
\hline
\end{tabular}


Anions by Ion Chromatography - Quality Control

Environmental Science Laboratory

\begin{tabular}{|c|c|c|c|c|c|c|c|c|c|c|}
\hline Analyte & Result & $\begin{array}{c}\text { orting } \\
\text { Limit }\end{array}$ & Units & $\begin{array}{l}\text { Spike } \\
\text { Level }\end{array}$ & $\begin{array}{l}\text { Source } \\
\text { Result }\end{array}$ & \%REC & $\begin{array}{l}\text { \%REC } \\
\text { Limits }\end{array}$ & RPD & $\begin{array}{l}\text { RPD } \\
\text { Limit }\end{array}$ & Notes \\
\hline
\end{tabular}

Batch 8F17009 - 1:1 Water Extract (IC)

\begin{tabular}{|c|c|c|c|c|c|c|c|c|}
\hline \multirow{2}{*}{$\begin{array}{l}\text { Blank (8F17009-BLK1) } \\
\text { Fluoride }\end{array}$} & \multicolumn{8}{|c|}{ Prepared: 06/17/08 Analyzed: 06/19/08 } \\
\hline & $<0.200$ & 0.200 & ug/g wet & & & & & \\
\hline Chloride & $<0.500$ & 0.500 & $"$ & & & & & \\
\hline Nitrite & $<1.00$ & 1.00 & $"$ & & & & & \\
\hline Bromide & $<1.00$ & 1.00 & $"$ & & & & & \\
\hline Nitrate & $<1.00$ & 1.00 & $"$ & & & & & \\
\hline Sulfate & $<1.50$ & 1.50 & " & & & & & \\
\hline Phosphate & $<1.50$ & 1.50 & $"$ & & & & & \\
\hline LCS (8F17009-BS1) & \multicolumn{8}{|c|}{ Prepared: 06/17/08 Analyzed: 06/19/08 } \\
\hline Fluoride & 2.09 & 0.200 & ug/g wet & 2.010 & 104 & $80-120$ & & \\
\hline Chloride & 5.16 & 0.500 & $"$ & 5.025 & 103 & $80-120$ & & \\
\hline Nitrite & 9.64 & 1.00 & $"$ & 10.05 & 96.0 & $80-120$ & & \\
\hline Bromide & 10.2 & 1.00 & $"$ & 10.05 & 102 & $80-120$ & & \\
\hline Nitrate & 10.4 & 1.00 & $"$ & 10.05 & 103 & $80-120$ & & \\
\hline Sulfate & 15.2 & 1.50 & $"$ & 15.08 & 101 & $80-120$ & & \\
\hline Phosphate & 15.0 & 1.50 & " & 15.08 & 99.2 & $80-120$ & & \\
\hline Duplicate (8F17009-DUP1) & \multicolumn{3}{|c|}{ Source: 0806012-01 } & \multicolumn{5}{|c|}{ Prepared: 06/17/08 Analyzed: 06/19/08 } \\
\hline Fluoride & $<2.07$ & 2.07 & ug/g dry & & & & & 20 \\
\hline Chloride & $<5.17$ & 5.17 & $"$ & & & & & 20 \\
\hline Nitrite & $<10.3$ & 10.3 & $"$ & & & & & 20 \\
\hline Bromide & $<10.3$ & 10.3 & " & & & & & 20 \\
\hline Nitrate & $<10.3$ & 10.3 & $"$ & & & & & 20 \\
\hline Sulfate & 48.1 & 15.5 & $"$ & & & & 7.80 & 20 \\
\hline Phosphate & $<15.5$ & 15.5 & $"$ & & & & & 20 \\
\hline
\end{tabular}


Anions by Ion Chromatography - Quality Control

Environmental Science Laboratory

\begin{tabular}{|lrrrrrrrrrrr}
\hline & & Reporting & & Spike & Source & & \%REC & & RPD & & \\
Analyte & Result & Limit & Units & Level & Result & \%REC & Limits & RPD & Limit & Notes \\
\hline
\end{tabular}

Batch 8F17009 - 1:1 Water Extract (IC)

Post Spike (8F17009-PS1)

Source: 0806012-01 Prepared: 06/17/08 Analyzed: 06/19/08

Fluoride

$\begin{array}{llllll}0.86 & \text { ug/mL } & 0.769 & 0.019 & 109 & 75-125\end{array}$

Chloride

Nitrite

Bromide

Nitrate

Sulfate

2.3

2.3

3.68

3.85

4.55

10.1

Phosphate

5.71

$\begin{array}{lllll}\text { " } & 1.92 & 0.288 & 105 & 75-125\end{array}$

\begin{tabular}{lllll}
$"$ & 1.92 & 0.288 & 105 & $75-125$ \\
\hline & 3.85 & & 95.7 & $75-125$
\end{tabular}

$\begin{array}{lllll} & 3.85 & \text { ND } & 95.7 & 75-125\end{array}$

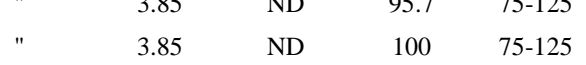

$\begin{array}{ccccc}" & 3.85 & \text { ND } & 100 & 75-125 \\ & 3.85 & 0.334 & 110 & 75-125\end{array}$

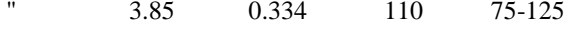

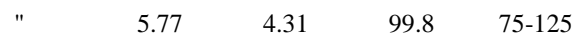

" 5.77

ND $\quad 98.9 \quad 75-125$




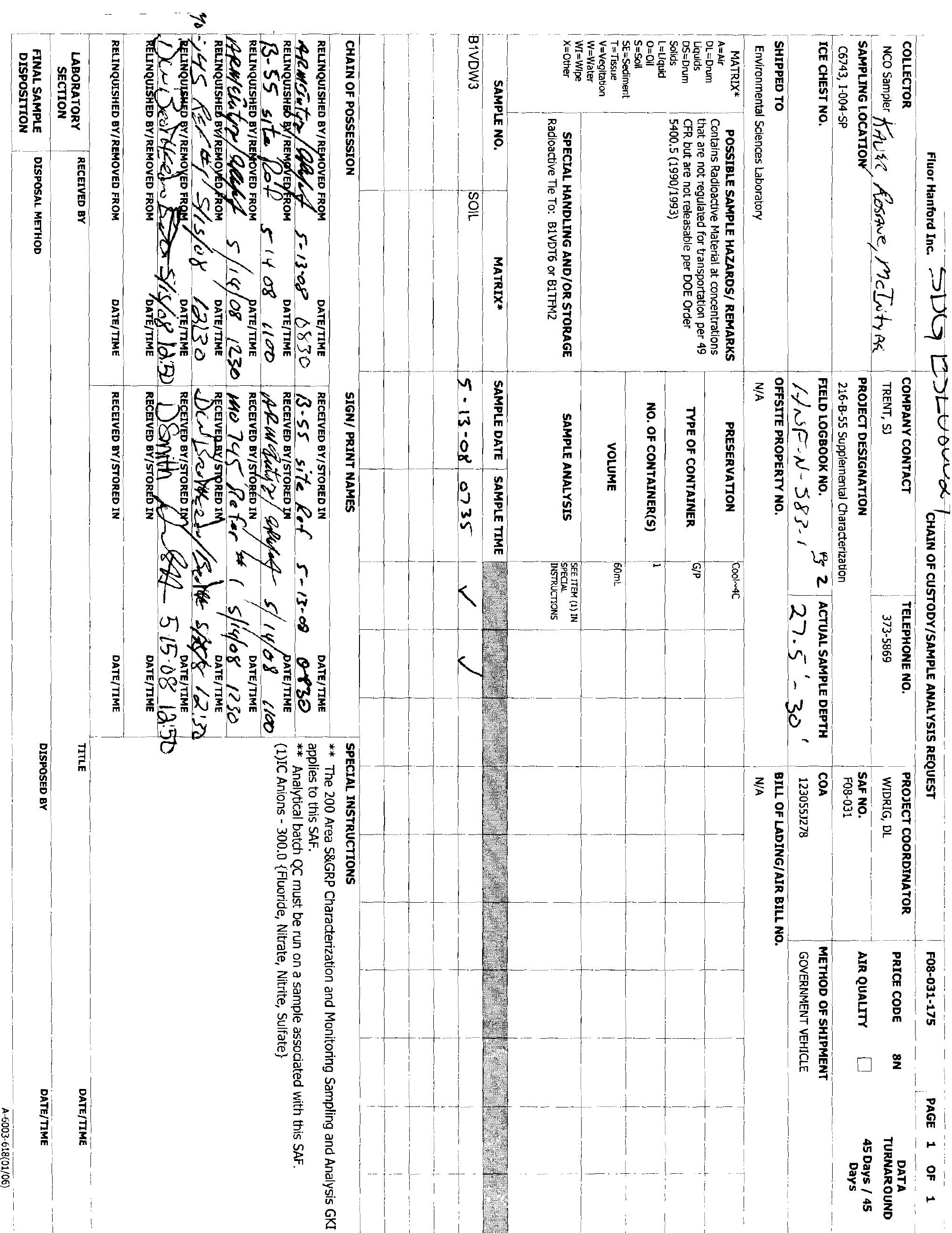

